\title{
The epidemiological characteristics and profile of drug-resistant tuberculosis among children with tuberculosis in Sichuan, China, 2015-2018: A retrospective study
}

\author{
Yuanhong Xu \\ Chengdu Public Health Clinical Center \\ Qingfeng Li \\ Chengdu Public Health Clinical Center \\ Ma Zhu \\ CDPHCC \\ Xueqi Wu \\ Sichuan University \\ Dongmei Wang \\ CDPHCC \\ Jia Luo \\ CDPHCC \\ Yingjie Li \\ CDPHCC \\ Jing Zhong \\ CDPHCC \\ Peibin Zeng ( $\sim$ zengpeibin@live.cn ) \\ Sichuan University
}

Research Article

Keywords: drug-resistant tuberculosis, child, Sichuan, Tibetan

Posted Date: July 13th, 2020

DOl: https://doi.org/10.21203/rs.3.rs-40972/v1

License: (1) This work is licensed under a Creative Commons Attribution 4.0 International License. Read Full License

Version of Record: A version of this preprint was published at Medicine on October 23rd, 2020. See the published version at https://doi.org/10.1097/MD.0000000000022608. 


\section{Abstract}

Background: The aim of this study was to investigate the epidemiological characteristics and profile of drug-resistant tuberculosis (DR-TB) among children with TB in Sichuan province of China.

Methods: From January 2015 to December 2018, microbiological culture-confirmed child TB cases (aged<15 years old) were enrolled retrospectively. Epidemiological and clinical information from these cases, and the drug susceptibility testing (DST) results of the isolates were collected and analyzed.

Results: Of 317 culture-confirmed child TB cases, 16.7\% (53/317) were aged under 5 years old. $54.9 \%$ were Tibetans, and $31.9 \%$ had clear history of contact with TB patients. More than half (53.9\%) weren't vaccinated by Calmette-Guérin bacillus (BCG). 30\% $(n=95)$ were diagnosed as severe TB, and $92.4 \%(n=293)$ were new cases. The ratio of severe TB in BCG vaccinated group was significant lower than that observed in unvaccinated group $(p<0.01)$. Significantly higher proportion of severe TB among Tibetans than Han child TB cases was observed in BCG unvaccinated group $(p<0.01)$. The overall rate of DR-TB in this study was $24.3 \%$ (77/317) and 17 multidrug-resistant tuberculosis (MDR-TB) cases were identified with rate of MDR-TB at 5.4\% (17/317). No XDR case was found. 13 out of 17 MDR-TB cases (76.4\%) were Tibetan children. The ratio of any resistance to four first-line drugs identified were: INH, $15.5 \%$; RIF, 9.1\%; EMB, $0.6 \%$ and SM, $6.0 \%$, respectively. More than half of MDR patterns were resistant to INH+RIF (9/17), followed by at least resistance to INH+RIF+SM $(n=7)$.

Conclusions: This was the first investigation on the epidemiological characteristics and profiles of DR-TB among child TB cases in Southwest of China. Our findings indicated a potentially high risk of TB infection to Tibetan children in the concentrated Tibetan communities of Sichuan.

\section{Background}

Known as an infectious disease with global distribution, Tuberculosis (TB) is one of major threats to public health with the leading causes of death [1]. Recent years, there is an increasing focus on the TB in children, previously neglected, to achieve the goal of zero childhood TB death [2]. Children with TB accounted for an estimated 11\% of the global burden of disease in 2018 [3], while more attentions have been paid to children affected by drug resistant TB (DR-TB), with an estimation of 25000 children developing multidrug-resistant TB (MDR-TB) and 1200 bearing extensively drug-resistant TB (XDR-TB) in 2014 alone [4]. Still, the visibility of epidemic and clear profile of drug-resistant (DR) among children with TB are both limited by the low sensitivity of TB diagnostic methods and the poor availability of specimens from children for microbiological confirmation and for drug susceptibility testing (DST) [5].

China has been ranked second after India on the list of estimated epidemiological burden for 30 high TB burden countries, with estimated 866,000 new cases in 2018 [3]. Limited studies describing the epidemic status and profile of DR in children with TB, indicated a gap between the number of estimated cases and reported cases [6], and relatively high proportions of MDR-TB among child TB cases in Shandong province (6.9\%) [6], Wuhan city (7.4\%) [7] and Chongqing city (6.5\%) [8]. Sichuan province is located in Southwest China with high diversity of ethnicities including the second largest Tibetan community after Tibet in China. The minority areas in Western Sichuan were reported to be with high incidence of TB [9]. However, there is still lack of data on demographic characteristics and profile of DR among children with TB in Sichuan province, nor on child TB Tibetans residing in Tibetan concentrated communities. In this study, we conducted a comprehensive and updated retrospective investigation on the demographic characteristics and profile of DR among child TB cases in Sichuan province, which is expected to provide data supporting on TB control and treatment for children with TB.

\section{Methods}

\section{Study site}

Chengdu (the capital city of Sichuan) public health clinic center (CDPHCC) is the largest regional TB specialty clinic, receiving TB patients from 21 cities and area of Sichuan province. Annually, approximated 20\% 30\% TB patients presenting at CDPHCC were 


\section{Cases and data collection}

From January 2015 to December 2018, 317 culture-confirmed pediatric TB cases (aged < 15 years old) presenting at Chengdu Public Health Clinical Center (CDPHCC), were included in this study ( $₫ 3 \%$ of overall culture-confirmed TB cases presenting at CDPHCC during the study period). Epidemiological and clinical information for cases including: age, gender, ethnicity, place of residence, history of contact with TB patients, BCG vaccination status, TB treatment history, and site of TB and disease severity were collected. According to the definitions recommended by WHO, TB new cases were defined as people who had never received anti-TB treatment, or been treated for less than one month, and retreatment cases were defined as those who had previously been treated at least one months or above.Severe TB was defined as TB disease with uncontrolled, disseminated, or complicated clinical syndromes $[10,11]$. Otherwise, cases were categorized into non-severe group.

TB confirmatory isolation cultures and identification for Mycobacterium tuberculosis (MTB)

Specimens including: sputum, flushing fluid from fiberoptic bronchoscopy, cerebrospinal fluid, hydrothorax and ascites, were collected for TB confirmatory isolation culture. The specimens were pre-treated to be decontaminated and homogenized following the laboratory guideline from WHO [12], and then underwent isolation culture by a semi-automated Mycobacterium rapid incubator (MGIT960; Becton Dickinson, Sparks, MD), following the protocol given by the manufacturer. The initial positive isolates were then tested by smear microscopy for acid-fast bacilli (AFB). AFB positive isolates were further cultured by thiophene-2carboxylic hydrazine (TCH) and p-nitrobenzoic acid (PNB) differential mediums to identify for MTB [13].

\section{Culture-based drug susceptibility testing (DST) and drug resistance definitions}

The drug susceptibility testing to 10 anti-tuberculosis drugs were performed (one isolate per case), using proportion method on Lowenstein-Jenson medium [14]. The 10 drugs and their concentrations on solid medium were: Rifampicin (RIF, $40 \mu \mathrm{g} / \mathrm{mL}$ ), isoniazid ( $\mathrm{INH}, 0.2 \mu \mathrm{g} / \mathrm{mL})$, streptomycin (SM, $4 \mu \mathrm{g} / \mathrm{mL})$, ethambutol (EMB, $2 \mu \mathrm{g} / \mathrm{mL})$, ofloxacin (OFX, $2 \mu \mathrm{g} / \mathrm{mL})$, levofloxacin (LFX, $2 \mu \mathrm{g} / \mathrm{mL}$ ), moxifloxacin (MFX, $2 \mu \mathrm{g} / \mathrm{mL}$ ), amikacin (AMK, $40 \mu \mathrm{g} / \mathrm{mL}$ ), kanamycin $(\mathrm{KM}, 30 \mu \mathrm{g} / \mathrm{mL}$ ) and capreomycin (CPM, $40 \mu \mathrm{g} / \mathrm{mL}$ ), respectively. MTB standard strain-H37Rv (sensitive to all drugs), provided by Sichuan Center for Disease Control and Prevention, was used as control in each batch of testing.

According to the definitions of TB drug resistance types from WHO[15], multidrug-resistant tuberculosis (MDR-TB) is defined as MTB with resistance to at least INH and RIF; Extensively drug-resistant tuberculosis (XDR-TB) is defined as MTB with resistance to at least four of core anti-TB drugs as: INH and RIF, in addition to any of the fluoroquinolones (such as OFX or MFX) and to at least one of three injectable second-line drugs (AMK, CPM or KM).

\section{Statistical analysis}

The statistical analysis was conducted using software SAS (SAS, Windows 9.4, SAS Institute, Cary, NC; 2016). Chi-square or Fisher's exact tests were performed on cases' demographic categories and drug-resistant status. $\mathrm{P}<0.05$ was considered statistically significant.

\section{Ethics statement}

This study was approved by the ethic committee of CDPHCC. Since this was a retrospective analysis, and patient records were anonymized and deidentified before analysis, the written consents from the patients' guardians were waived.

\section{Results}

The characteristics of culture-confirmed child TB cases

Of 317 culture-confirmed child TB cases, more than half (54.6\%) were aged between 12 and 15 years old (Table 1). Only 16.7\% $(53 / 317)$ were aged under 5 years old. The gender ratio was comparable, and $54.9 \%$ cases were Tibetans, followed by Han (30.6\%), Yi (11.4\%) and others (3.2\%) by ethnicity. 89.3\% $(n=283)$ cases lived in the rural area, and $31.9 \%$ had clear history of 
contact with TB patients. More than half of child TB cases (53.9\%) weren't vaccinated by Calmette-Guérin bacillus (BCG). 30\% (n = 95) were diagnosed as severe TB and $92.4 \%(n=293)$ were new cases. 
Table 1

The characteristics of culture-confirmed child TB cases in Sichuan from Jan 2015 to Dec 2018.

\begin{tabular}{|c|c|c|c|}
\hline Characteristics & $\begin{array}{l}\text { Cases with any resistance, total } \\
=77 \\
(n, \%)\end{array}$ & $\begin{array}{l}\text { Cases without resistance, total = } \\
240 \\
(n, \%)\end{array}$ & $\begin{array}{l}\text { Total }= \\
317 \\
(n, \%)\end{array}$ \\
\hline \multicolumn{4}{|l|}{ Age (years) } \\
\hline$<5$ & $12(22.6)$ & $41(77.4)$ & 53 (16.7) \\
\hline $5 \otimes 11$ & $22(24.2)$ & $69(75.8)$ & $91(28.7)$ \\
\hline $12 \bigotimes 15$ & $43(24.9)$ & $130(75.1)$ & $\begin{array}{l}173 \\
(54.6)\end{array}$ \\
\hline \multicolumn{4}{|l|}{ Gender } \\
\hline Male & $40(25.6)$ & 116(74.4) & $156(49.2)$ \\
\hline Female & $37(23.0)$ & 124(77.0) & $161(50.8)$ \\
\hline \multicolumn{4}{|l|}{ Ethnicity } \\
\hline Han & 23(23.7) & 74(76.3) & $97(30.6)$ \\
\hline Tibetan & $45(25.9)$ & $129(74.1)$ & $174(54.9)$ \\
\hline Yi & $5(13.9)$ & $31(86.1)$ & $36(11.4)$ \\
\hline Others & $4(40.0)$ & $6(60.0)$ & 10(3.2) \\
\hline \multicolumn{4}{|l|}{ Residential area } \\
\hline Urban & $8(23.5)$ & $26(76.5)$ & $34(10.7)$ \\
\hline Rural & $69(24.4)$ & 214(75.6) & 283(89.3) \\
\hline \multicolumn{4}{|c|}{$\begin{array}{l}\text { Known history of contact with TB } \\
\text { patients }\end{array}$} \\
\hline Yes & $28(27.7)$ & 73(72.3) & 101(31.9) \\
\hline Not clear & $49(22.7)$ & 167(77.3) & $216(68.1)$ \\
\hline \multicolumn{4}{|l|}{ BCG vaccination } \\
\hline Yes & $15(25.0)$ & $45(75.0)$ & $60(18.9)$ \\
\hline No & $41(24.0)$ & $130(76.0)$ & $171(53.9)$ \\
\hline Unknown & $21(24.4)$ & $65(75.6)$ & $86(27.1)$ \\
\hline \multicolumn{4}{|l|}{ Severe TB ${ }^{a}$} \\
\hline Yes & $21(22.1)$ & 74(77.9) & $95(30.0)$ \\
\hline No & $56(25.2)$ & $166(74.8)$ & $222(70.0)$ \\
\hline \multicolumn{4}{|c|}{ Treatment status ${ }^{b}$} \\
\hline New case & $67(22.9)$ & $226(77.1)$ & 293(92.4) \\
\hline Retreatment & $10(41.7)$ & 14(58.3) & $24(7.6)$ \\
\hline \multicolumn{4}{|c|}{ a, Severe TB was defined as TB disease with uncontrolled, disseminated, or complicated clinical syndromes } \\
\hline$b$, The ratio of $c$ & ong new & rthan that & $<0.05$ \\
\hline
\end{tabular}


All 317 MTB isolates from culture-confirmed child TB cases (one isolate per case) were tested to have valid DST results. Of these 317 isolates, 77 were identified to be with any resistance to all the 10 anti-tuberculosis drugs, while 240 (75.7\%) were fully sensitive (Table 1). The cases in both with and without resistance groups were similar in terms of their characteristics mentioned above, except between new and retreatment cases, where significantly higher proportion of cases with any resistance $(p<0.05)$ was found in retreatment group.

Severe TB versus BCG vaccination in different ethnicities

The reported BCG vaccinated $(n=60)$ and unvaccinated $(n=171)$ cases were selected and analyzed for the distribution of severe TB by BCG vaccination status in different ethnicities (Table 2). Higher proportion of Tibetans was observed in BCG unvaccinated group, compared to other ethnicities $(p<0.01)$. The overall ratio of severe

Table 2

The distribution of severe TB by BCG vaccination status in different ethnicities.

\begin{tabular}{|lllll|}
\hline Severe TB $^{\mathrm{a}}$ & Yes $^{\mathrm{b}}$ & No & Total (n, \%) \\
\hline BCG vaccinated & Tibetan $(\mathrm{n}, \%)$ & $2(16.7)$ & $10(83.3)$ & $12(20.0)$ \\
\hline Han $(\mathrm{n}, \%)$ & $6(14.3)$ & $36(85.7)$ & $42(70.0)$ \\
\hline Yi(n, \%) & $1(25.0)$ & $3(75.0)$ & $4(6.7)$ \\
\hline Others $(\mathrm{n}, \%)$ & $1(50.0)$ & $1(50.0)$ & $2(3.3)$ \\
\hline Total $(\mathrm{n}, \%)$ & $10(16.7)^{\mathrm{c}}$ & $50(83.3)$ & $60(26.0)$ \\
\hline BCG unvaccinated & Tibetan $(\mathrm{n}, \%)$ & $50(45.5)^{\mathrm{d}}$ & $60(54.5)$ & $110(64.3)$ \\
\hline Han $(\mathrm{n}, \%)$ & $5(17.2)^{\mathrm{d}}$ & $24(82.8)$ & $29(17.0)$ \\
\hline Yi(n, \%) & $11(44.0)$ & $14(56.0)$ & $25(14.6)$ \\
\hline Others $(n, \%)$ & $1(14.3)$ & $6(85.7)$ & $7(4.1)$ \\
\hline Total $(n, \%)$ & $67(39.2)^{\mathrm{c}}$ & $104(60.8)$ & $171(74.0)$ \\
\hline
\end{tabular}

a, Severe TB was defined as TB disease with uncontrolled, disseminated, or complicated clinical syndromes.

$b$, Despite of BCG vaccination, overall ratio of severe TB among Tibetans was significantly higher $(p<0.01)$ than that from Han. (Tibetan: 52/77, 67.5\% V.S. Han: 11/77, 14.3\%); For Tibetans, the severe TB rate of BCG unvaccinated group was higher than BCG vaccinated group, but not significantly. (45.5\% V.S. $16.7, p=0.11)$

c, The overall ratio of severe TB from BCG vaccinated group was significant lower than that from unvaccinated group, $p<0.01$

d, Severe TB rate of Tibetans was significant higher than that in the Han, within BCG unvaccinated group, $p<0.01$

TB from BCG vaccinated group was significant lower than that from unvaccinated group $(p<0.01)$.

Despite of BCG vaccination, overall ratio of severe TB among Tibetans was significantly higher $(p<0.01)$ than that from Han. (Tibetan: 52/77, 67.5\% V.S. Han: 11/77, 14.3\%). For Tibetans, severe TB rate of BCG vaccinated group was lower than BCG unvaccinated group, but not significantly $(p=0.11)$; While the severe TB rate was similar between BCG vaccinated and unvaccinated group among Han cases. Furthermore, significantly higher proportion of severe TB among Tibetans than Han child TB cases was observed in BCG unvaccinated group $(p<0.01)$.

The profile of drug resistance

The overall rate of DR-TB in this study was $24.3 \%$ (77/317). The profile of drug resistance for 317 isolates was displayed in Fig. 1. For 293 new and 24 retreatment cases, the rates of DR-TB were $22.9 \%$ and $41.7 \%$, respectively (Table 3 ). The ratio of any resistance to four first-line drugs identified among 317 isolates were: INH, 15.5\%; RIF, 9.1\%; EMB, 0.6\% and SM, 6.0\%, respectively. 24 new cases were found to have mono-resistant to 5 anti-TB drugs, where only one retreatment case had mono-resistant to INH. 
Table 3

Profiles of drug resistance among Mycobacterium tuberculosis isolates in Sichuan from Jan 2015 to Dec 2018 . $^{{ }^{\prime}}$

\begin{tabular}{|c|c|c|c|}
\hline Drug resistance & New cases, no. (\%), total $=293$ & Retreatment, no. $(\%)$, total $=24$ & Total $=317$, no. $(\%)$ \\
\hline Any resistance ${ }^{b}$ & $67(22.9)$ & $10(41.7)$ & $77(24.3)$ \\
\hline \multicolumn{4}{|l|}{ Any resistance to first-line drug } \\
\hline INH & $40(13.7)$ & $9(37.5)$ & 49 (15.5) \\
\hline RIF & $22(7.5)$ & $7(29.2)$ & $29(9.1)$ \\
\hline EMB & $1(0.3)$ & $1(4.2)$ & $2(0.6)$ \\
\hline SM & $16(5.5)$ & $3(12.5)$ & $19(6.0)$ \\
\hline Mono-resistant & $42(14.3)$ & $1(4.2)$ & $43(13.7)$ \\
\hline INH & $24(8.2)$ & $1(4.2)$ & $25(7.9)$ \\
\hline RIF & $11(3.8)$ & 0 & $11(3.5)$ \\
\hline SM & $5(1.7)$ & 0 & $5(1.6)$ \\
\hline CPM & $1(0.3)$ & 0 & $1(0.3)$ \\
\hline $\mathrm{KM}$ & $1(0.3)$ & 0 & $1(0.3)$ \\
\hline Multi-drug resistant (MDR) ${ }^{b}$ & $10(3.4)$ & $7(29.2)$ & $17(5.4)$ \\
\hline $\mathrm{INH}+\mathrm{RIF}$ & $5(1.7)$ & $4(16.7)$ & $9(2.8)$ \\
\hline At least to INH + RIF + SM & $4(1.4)$ & $3(12.5)$ & $7(2.2)$ \\
\hline Other forms of poly-resistant & $15(5.1)$ & $2(8.3)$ & $17(5.4)$ \\
\hline At least to OFX + MFX & $8(2.7)$ & $1(4.2)$ & $9(2.8)$ \\
\hline At least to INH/RIF + SM & $7(2.4)$ & 0 & $7(2.2)$ \\
\hline
\end{tabular}

Figure 1. The profile of drug resistant of $\mathbf{3 1 7}$ mycobacterium tuberculosis isolates from culture-confirmed TB children in Sichuan from Jan 2015 to Dec 2018. Abbreviations: RIF, rifampicin; INH, isoniazid; SM, streptomycin; EMB, ethambutol; OFX, ofloxacin; MFX, moxifloxacin; LFX, levofloxacin; AMK, amikacin; KM, kanamycin; and CPM, capreomycin.

17 MDR cases were identified with rate of MDR-TB at 5.4\% (17/317), in which the MDR TB rate of retreatment cases $(29.2 \%, 7 / 24)$ was significant higher $(p<0.01)$ than that from new cases $(3.4 \%, 10 / 293)$. More than half of MDR patterns were resistant to INH + RIF $(n=9)$, followed by at least resistance to INH + RIF + SM $(n=7)$. Among seventeen other forms of poly-resistant cases, patterns of at least resistance to OFX + MFX $(n=9)$ and to INH/RIF + SM $(n=7)$ were most commonly observed. No XDR case was found in this study.

The characteristics of 17 MDR child TB cases

With regard to 17 MDR cases, most were Tibetans (13/17) (Table 4). The age distribution was from 2 to 14 years old, similar to that observed from 317 culture-confirmed cases. Only two were reported to be vaccinated by BCG, and 2 out of 17 MDR cases was diagnosed as pulmonary TB with tuberculous meningitis. 9 cases had clear history contact to their families, especially the patient ID 4, whose father was identified as DR-TB. More than half of the MDR cases $(n=9)$ were previously received TB treatment with similar regimens and doses (INH, RIF, EMB and pyrazinamide), varied by the duration of treatment between 1 month 20 days to 16 months. 
Table 4

The characteristics of multi-drug resistant (MDR) cases ( $n=17)$ in Sichuan province, Jan 2015 to Dec 2018.

\begin{tabular}{|c|c|c|c|c|c|c|c|c|c|c|}
\hline ID & Gender & $\begin{array}{l}\text { Age } \\
\text { in } \\
\text { years }\end{array}$ & Ethnicity & $\begin{array}{l}\text { BCG } \\
\text { vaccination }\end{array}$ & $\begin{array}{l}\text { Severe } \\
\text { TB }^{\text {a }}\end{array}$ & $\begin{array}{l}\text { History of } \\
\text { contact with } \\
\text { tuberculosis } \\
\text { patients } \\
\text { (Source) }\end{array}$ & $\begin{array}{l}\text { Forms } \\
\text { of } \\
\text { MDR } \\
b\end{array}$ & $\begin{array}{l}\text { Previous } \\
\text { TB } \\
\text { Treatment }\end{array}$ & $\begin{array}{l}\text { Previous } \\
\text { treatment } \\
\text { regimen c }\end{array}$ & $\begin{array}{l}\text { Duration } \\
\text { of } \\
\text { previous } \\
\text { treatment }\end{array}$ \\
\hline 1 & Male & 10 & Han & unknown & No & Yes (Father) & $\begin{array}{l}\text { INH + } \\
\text { RFP + } \\
\text { SM }\end{array}$ & Yes & $\begin{array}{l}\text { INH } \\
(0.3 \mathrm{~g}), \\
\text { RFP } \\
(0.45 \mathrm{~g}), \\
\text { EMB } \\
(0.45 \mathrm{~g}) \\
\text { and PZA } \\
(0.625 \mathrm{~g})\end{array}$ & $\begin{array}{l}1 \text { months } \\
20 \text { days }\end{array}$ \\
\hline 2 & Female & 12 & Tibetan & unknown & No & Yes (Mother) & $\begin{array}{l}\text { INH + } \\
\text { RFP }\end{array}$ & No & / & / \\
\hline 3 & Female & 14 & Tibetan & unknown & No & Not clear & $\begin{array}{l}\text { INH+ } \\
\text { RFP }\end{array}$ & Yes & $\begin{array}{l}\text { Not } \\
\text { available }\end{array}$ & 2 months \\
\hline 4 & Male & 11 & $\mathrm{Yi}$ & No & No & $\begin{array}{l}\text { Yes (Father, } \\
\text { who was } \\
\text { identified as } \\
\text { DR-TB) }\end{array}$ & $\begin{array}{l}\text { INH + } \\
\text { RFP + } \\
\text { SM + } \\
\text { EMB } \\
+ \text { OFX } \\
+ \text { LFX } \\
+ \text { MFX }\end{array}$ & Yes & $\begin{array}{l}\text { INH } \\
(0.3 \mathrm{~g}), \\
\text { RFP } \\
(0.45 \mathrm{~g}) \text {, } \\
\text { EMB } \\
(0.5 \mathrm{~g}) \\
\text { and PZA } \\
(0.75 \mathrm{~g})\end{array}$ & $\begin{array}{l}2 \text { months } \\
12 \text { days }\end{array}$ \\
\hline 5 & Female & 10 & Tibetan & No & No & Yes (Sister) & $\begin{array}{l}\text { INH + } \\
\text { RFP + } \\
\text { OFX + } \\
\text { LFX + } \\
\text { MFX }\end{array}$ & No & / & / \\
\hline 6 & Male & 7 & Tibetan & No & No & Not clear & $\begin{array}{l}\text { INH + } \\
\text { RFP + } \\
\text { SM + } \\
\text { OFX + } \\
\text { LFX + } \\
\text { MFX }\end{array}$ & No & / & / \\
\hline 7 & Female & 12 & Tibetan & unknown & No & Yes (Father) & $\begin{array}{l}\text { INH+ } \\
\text { RFP }\end{array}$ & Yes & $\begin{array}{l}\text { INH } \\
(0.2 \mathrm{~g}), \\
\text { RFP } \\
(0.3 \mathrm{~g}) \\
\text { and PZA } \\
(1 \mathrm{~g})\end{array}$ & 9 months \\
\hline 8 & Female & 14 & Tibetan & unknown & No & Not clear & $\begin{array}{l}\text { INH+ } \\
\text { RFP + } \\
\text { SM+ } \\
\text { EMB }\end{array}$ & No & / & / \\
\hline 9 & Male & 7 & Tibetan & No & No & Yes (Cousin) & $\begin{array}{l}\text { INH + } \\
\text { RFP + } \\
\text { SM }\end{array}$ & No & / & / \\
\hline
\end{tabular}

a, Severe TB was defined as TB disease with uncontrolled, disseminated, or complicated clinical syndromes.

b, Abbreviations: RIF, rifampicin; INH, isoniazid; SM, streptomycin; EMB, ethambutol; OFX, ofloxacin; LFX, levofloxacin; MFX, moxifloxacin.

c, PZA, Pyrazinamide; Dose annotations: oral administration once daily for INH, EMB and PZA; Intravenous drip once daily for RFP.

d, Diagnosed as pulmonary TB with tuberculous meningitis 


\begin{tabular}{|c|c|c|c|c|c|c|c|c|c|c|}
\hline ID & Gender & $\begin{array}{l}\text { Age } \\
\text { in } \\
\text { years }\end{array}$ & Ethnicity & $\begin{array}{l}\text { BCG } \\
\text { vaccination }\end{array}$ & $\begin{array}{l}\text { Severe } \\
\text { TB }^{a}\end{array}$ & $\begin{array}{l}\text { History of } \\
\text { contact with } \\
\text { tuberculosis } \\
\text { patients } \\
\text { (Source) }\end{array}$ & $\begin{array}{l}\text { Forms } \\
\text { of } \\
\text { MDR } \\
b\end{array}$ & $\begin{array}{l}\text { Previous } \\
\text { TB } \\
\text { Treatment }\end{array}$ & $\begin{array}{l}\text { Previous } \\
\text { treatment } \\
\text { regimen }\end{array}$ & $\begin{array}{l}\text { Duration } \\
\text { of } \\
\text { previous } \\
\text { treatment }\end{array}$ \\
\hline 10 & Female & 2 & Tibetan & No & No & $\begin{array}{l}\text { Yes } \\
\text { (Grandfather) }\end{array}$ & $\begin{array}{l}\text { INH+ } \\
\text { RFP }\end{array}$ & No & / & / \\
\hline 11 & Male & 6 & Tibetan & No & No & Not clear & $\begin{array}{l}\text { INH+ } \\
\text { RFP }\end{array}$ & No & / & / \\
\hline 12 & Male & 10 & Tibetan & No & No & Not clear & $\begin{array}{l}\text { INH+ } \\
\text { RFP }\end{array}$ & No & / & / \\
\hline 13 & Male & 12 & Han & Yes & No & Not clear & $\begin{array}{l}\text { INH+ } \\
\text { RFP + } \\
\text { SM }\end{array}$ & Yes & $\begin{array}{l}\text { INH } \\
(0.3 \mathrm{~g}), \\
\text { RFP } \\
(0.45 \mathrm{~g}) \\
\text { and EMB } \\
(0.75 \mathrm{~g})\end{array}$ & 7 months \\
\hline 14 & Male & 14 & Tibetan & unknown & Yes $^{d}$ & Not clear & $\begin{array}{l}\text { INH+ } \\
\text { RFP }\end{array}$ & Yes & $\begin{array}{l}\text { INH } \\
(0.3 \mathrm{~g}) \text {, } \\
\text { RFP } \\
(0.45 \mathrm{~g}) \text {, } \\
\text { EMB } \\
(0.5 \mathrm{~g}) \\
\text { and PZA } \\
(0.75 \mathrm{~g})\end{array}$ & 3 months \\
\hline 15 & Male & 1 & Han & Yes & No & Not clear & $\begin{array}{l}\text { INH+ } \\
\text { RFP }\end{array}$ & Yes & $\begin{array}{l}\text { INH } \\
(0.3 \mathrm{~g}) \text {, } \\
\text { RFP } \\
(0.3 \mathrm{~g}) \text {, } \\
\text { EMB } \\
(0.5 \mathrm{~g}) \\
\text { and PZA } \\
(0.75 \mathrm{~g}) \text {, } \\
3 \\
\text { months/ } \\
\text { INH } \\
(0.3 \mathrm{~g}) \\
\text { and RFP } \\
(0.45 \mathrm{~g}) \text {, } \\
13 \\
\text { months }\end{array}$ & $\begin{array}{l}16 \\
\text { months }\end{array}$ \\
\hline 16 & Male & 14 & Tibetan & unknown & No & Yes (Mother) & $\begin{array}{l}\text { INH+ } \\
\mathrm{RFP}+ \\
\text { SM }\end{array}$ & No & / & / \\
\hline 17 & Male & 14 & Tibetan & unknown & Yes $^{d}$ & Yes (Mother) & $\begin{array}{l}\text { INH+ } \\
\text { RFP }\end{array}$ & Yes & $\begin{array}{l}\text { Not } \\
\text { available }\end{array}$ & 2 months \\
\hline \multicolumn{11}{|c|}{ a, Severe TB was defined as TB disease with uncontrolled, disseminated, or complicated clinical syndromes. } \\
\hline \multicolumn{11}{|c|}{$\begin{array}{l}\text { b, Abbreviations: RIF, rifampicin; INH, isoniazid; SM, streptomycin; EMB, ethambutol; OFX, ofloxacin; LFX, levofloxacin; MFX, } \\
\text { moxifloxacin. }\end{array}$} \\
\hline \multicolumn{11}{|c|}{$\begin{array}{l}\text { c, PZA, Pyrazinamide; Dose annotations: oral administration once daily for INH, EMB and PZA; Intravenous drip once daily for } \\
\text { RFP. }\end{array}$} \\
\hline
\end{tabular}

\section{Discussion}

Based on the data of a four-year (2015-2018) retrospective analysis, our study provided a comprehensive review of epidemiological characteristic and drug resistance profile of child TB cases in Sichuan province. During the study period, the 
proportion of culture-confirmed child TB cases ( $<15$ years old) was only about $3 \%$ of all culture confirmed TB cases in CDPHCC. This proportion was higher than the previous study in Shandong province, where the rate of culture-confirmed child TB cases among all new TB cases was 1.3\% [6], but lower than the estimated proportion of child TB in China, which is larger than 5\% [16]. In addition, young children $<5$ years of age accounted $16.7 \%$ of child TB cases here, and the rate was far lower than that from the WHO data, predicting that $58 \%$ of total child TB cases are from the group of young children ( $<5$ years old) [16]. It is commonly recognized the underreporting for child TB cases worldwide and especially in low- and middle-income countries and area[6, 16], which probably due to two reasons: 1) the pool availability of specimens from children with TB for microbiological diagnosis. Child TB cases are more likely to have paucibacillary disease and the young children ( $<5$ years old ) cannot expectorate sputum [17]. As a matter of course, many child TB cases are diagnosed without microbiological confirmation; 2) the low sensitivity of microbiological tests for child TB cases[5], which is believed to further enlarge the gap between number of expected cases and reported cases $[16,18]$.

In our study, more than half of child TB cases were Tibetans (54.9\%). It should be noticed that Tibetans concurrently accounted approximate $20 \%$ of total TB case in CDPHCC, indicating the increasing proportion of child TB cases among Tibetans, in accordance with the perspective that children are more likely to develop into TB disease than adult after infection with TB [4]. There is still lack of study on the Tibetan children with TB. Former investigation in Tibet, described a significant higher incidence of TB infection in Tibetans, compared to the Han in general population [19]. The concentrated communities for Tibetans in Sichuan are mostly the least developed rural area [20], and the influence of poverty is reported to be associated with increased risk of being exposed to TB of being infected, developing disease and severe outcomes for children [21]. Consequently, there is no surprise to find higher ratio of severe TB among child TB cases of Tibetans. Further, children in contact with TB patients are known as high risk group for TB infection [22]. 31.9\% child TB cases were with clear history of contact with TB patients in our study. The actual risk of children to TB infection is expected to be even higher in concentrated Tibetan communities in Sichuan, not only because of the resource-limited settings and pool hygiene awareness [23], but also the increased susceptibility to TB for children and large potential transmission source such as latent TB under the iceberg [24]. Strengthening the recommended contact investigation to trace the TB source case-patients living close to young children [25], together with more effective diagnostic strategy for TB microbiological confirmation are urgent needed to better understand the incidence and risk of TB among children in this area.

Our finding agreed with the previous studies indicating the BCG vaccination could decrease the risk of severe TB [26, 27]. The BCG vaccination had demonstrated its power, especially herein the Tibetan child TB cases, of those whom the rate of severe TB from BCG vaccinated group was lower than that from BCG unvaccinated group. Unfortunately, the overall ratio of BCG un-vaccination for child TB cases was still high (53.9\%), and even higher in Tibetan child TB cases $(63.2 \%, 110 / 174)$. According to the national vaccination policy, BCG is one of basic vaccines for free and suggested to be vaccinated to neonates within 24 hours after birth. However, vaccination awareness is pool in some underdeveloped area [28]. Improving the vaccination rate of BCG, especially in concentrated Tibetan communities, could be a feasible way to reduce the proportion of severe child TB in Sichuan province.

The overall rates of DR-TB among culture confirmed child TB observed in our study was $24.3 \%$, and the rate was similar to the previous studies in Chinese Wuhan (28.4\%) [7] and Chongqing (20.9\%) [8], New Delhi (20.5\%)[29] and Mexico (26.7\%) [30], but higher than that observed in Korean (13.5\%) [31]; The overall rate of MDR-TB here was 5.4\%, which was slightly lower than those from Wuhan (7.4\%) [7] and Chongqing (6.5\%) [8], but higher than that in the U.S. (1.6\%) [32] and from a systematic review with global estimates of 97 studies on child TB cases at 4\% [5]. 45 out of 77 DR-TB (58.4\%) and 13 out of 17 MDR-TB cases (76.4\%) were Tibetan child TB cases, which could probably because of more contacts with TB patients for these children, who were reported to bear higher probability to have MDR-TB $[33,34]$.

The rates of DR-TB and MDR-TB among new child TB cases were $22.9 \%$ and $3.4 \%$ in our study. Compared to a similar study in Shandong [6], the rate of DR-TB of new cases was comparable (18.9\%), while the MDR-TB rate was lower than those observed from that study (6.9\%). Both rates of DR-TB and MDR-TB from new child TB cases were lower than that previous national DR-TB survey in China, where the proportions of DR-TB and MDR-TB cases were $34.2 \%$ and $5.7 \%$ among all the new TB cases, respectively [1]. If we assume the DR from new child TB cases were mostly the transmitted DR, the moderate proportions of DR-TB and MDR-TB within new cases could partly reflect the transmission status of DR and MDR-TB strains in Sichuan.

Page $10 / 14$ 
Compared to new cases, the significantly diminishing of mono-resistant and increasing of MDR among retreatment cases were identified in our study. Additionally, the proportions of resistance to four first-line drugs (INH, RIF, EMB and SM) from retreatment cases were all higher than that observed in new cases. These findings implied the potential development of drug resistant status following the anti-TB treatment among child TB cases in Sichuan. According to the Guidelines for the rational use of antibiotics in acute respiratory infection from Chinese National Health Commission, aminoglycosides are forbidden for children under 6 years old, and for children over 6 years old, which should be used with caution due to the ototoxicity and nephrotoxicity of these antibiotics [35]. So, it is no surprise that the resistance to anti-TB drugs of aminoglycosides such as AMK and KM, are barely found in our study. The proportions of fluoroquinolones (such as OFX, MFX and LFX) were identified to be higher than our previous investigation in adult TB cases in Sichuan [36]. Though various adverse effects have been reported[37, 38], fluoroquinolones are still considered as key components of current MDR-TB regimens[39]. The certain numbers of resistance to fluoroquinolones among new child TB cases are likely from the MDR-TB patients with fluoroquinolones treatment, which should be concerned for their impact on the circulation patterns of DR-TB strains in this area.

There are several limitations for our study. First, there is no follow-up result to monitor the ongoing development of DR following the anti-TB treatment, as well as the treatment outcomes. Our future effort will strive to conduct the follow-up study to evaluate the treatment regimens for child TB cases, helping on optimization of treatment strategy for child TB. Secondly, the lack of contact investigation may limit the power to accurately investigate the incidence of child TB in this area, which is urgent needed. Last, we don't have the genotypic information on the DR TB strains, which is important to draw a more comprehensive picture on the current status of circulation TB strains in this area.

\section{Conclusion}

In conclusion, this was the first investigation on the epidemiological characteristics and profiles of DR-TB among child TB cases in Southwest of China. Our findings shed light on indicating a potentially high risk for Tibetan children to TB infection in the concentrated Tibetan communities of Sichuan. Our results support continuing DR-TB surveillance as part of comprehensive TB control and treatment in China.

\section{Abbreviations}

$\mathrm{TB}=$ tuberculosis, $\mathrm{DR}=$ drug resistant, $\mathrm{MDR}$ = multidrug-resistant, $\mathrm{XDR}$ = extensively drug-resistant, $\mathrm{DST}$ = drug susceptibility testing, $\mathrm{CDPHCC}=$ Chengdu public health clinic center, $\mathrm{BCG}=$ Calmette-Guérin bacillus, MTB = Mycobacterium tuberculosis, AFB = acid-fast bacilli, $\mathrm{TCH}$ = thiophene-2-carboxylic hydrazine, $\mathrm{PNB}=$-nitrobenzoic acid, RIF = rifampicin, INH = isoniazid, SM = streptomycin, EMB = ethambutol, OFX = ofloxacin, LFX = levofloxacin, MFX = moxifloxacin, $\mathrm{AMK}=$ amikacin, $\mathrm{KM}=$ kanamycin, $\mathrm{CPM}=$ capreomycin, $\mathrm{PZA}=$ Pyrazinamide.

\section{Declarations}

\section{Ethics approval and consent to participate}

This study was approved by the ethic committee of CDPHCC. Since this was a retrospective analysis, and patient records were anonymized and deidentified before analysis, the written consents from the patients' guardians were waived.

\section{Consent for publication}

Not applicable.

\section{Availability of data and materials}

All data generated or analysed during this study are included in this published article.

\section{Competing interests}

Page 11/14 
The authors declare that they have no competing interests.

\section{Funding}

The study was supported by the Key Research and Development Project, Department of Science and Technology, Sichuan Province (Grant No. 2018SZ0212 and 2018JY0383); and the Fundamental Research Funds for the Central Universities; Project of Sichuan health and Family Planning Commission (Grant No. 18PJ015). The funders had no role in the study design, data collection and analysis, decision to publish, or preparation of the manuscript.

\section{Authors' contributions}

QL and PZ designed the study. YX and MZ collected the samples. YX and XW did the experiments. DW and JL helped on the investigation. $Y L, J Z$ and $P Z$ analyzed the data. $Y X$ and $P Z$ wrote the paper. All authors read and approved the final manuscript.

\section{Acknowledgements}

"Not applicable"

\section{References}

1. Zhao Y, Xu S, Wang L, Chin DP, Wang S, Jiang G, Xia H, Zhou Y, Li Q, Ou X, et al. National survey of drug-resistant tuberculosis in China. N Engl J Med. 2012;366(23):2161-70.

2. WHO. The roadmap for childhood TB: toward zero deaths. https://www.who.int/tb/publications/tb-childhoodroadmap/en/. Last access: 2019/10/27.

3. WHO. Global Tuberculosis R. 2019. https://www.who.int/tb/publications/global_report/en/. Last access: 2019/10/28.

4. Dodd PJ, Sismanidis C, Seddon JA. Global burden of drug-resistant tuberculosis in children: a mathematical modelling study. Lancet Infect Dis. 2016;16(10):1193-201.

5. Jenkins HE, Tolman AW, Yuen CM, Parr JB, Keshavjee S, Perez-Velez CM, Pagano M, Becerra MC, Cohen T. Incidence of multidrug-resistant tuberculosis disease in children: systematic review and global estimates. Lancet. 2014;383(9928):15729.

6. Tao NN, He XC, Zhang XX, Liu Y, Yu CB, Li HC. Drug-Resistant Tuberculosis among Children, China, 2006-2015. Emerg Infect Dis. 2017;23(11):1800-5.

7. Huang QL, KX, Yu ZX. Epidemiological and drug resistance analysis of 458 children with tuberculosis in Wuhan from 2013 to 2015. Pract Prev Med. 2017;24(12):1511-3.

8. Peng ZL, Ru X, Li QZ, Xing LL, Zhu CM. Risk factors of drug-resistant pediatric tuberculosis patients. CHINESE JOURNAL OF MICROECOLOGY. 2011;23(7):644-6.

9. Xia YL, Li YK, Wang DX, He JG, Zhang PR. Tuberculosis Epidemic Status in Sichuan Province During 2004 and 2014 and Trend Prediction. Journal of Preventive Medicine Information. 2015;31(12):946-9.

10. Seddon JA, Perez-Velez CM, Schaaf HS, Furin JJ, Marais BJ, Tebruegge M, Detjen A, Hesseling AC, Shah S, Adams LV, et al. Consensus Statement on Research Definitions for Drug-Resistant Tuberculosis in Children. J Pediatric Infect Dis Soc. 2013;2(2):100-9.

11. Wiseman CA, Gie RP, Starke JR, Schaaf HS, Donald PR, Cotton MF, Hesseling AC. A proposed comprehensive classification of tuberculosis disease severity in children. Pediatr Infect Dis J. 2012;31(4):347-52.

12. De Kantor NIKS, Frieden T, Laszlo A, Luelmo F, Norval P, et al. Laboratory services in tuberculosis control. In: WHO/TB/98.258. Geneva: World Health Organization; 1998.

13. Zhao YL. Procedures for tuberculosis laboratory testing. People's Health Press 1st edition. 2015.

Page $12 / 14$ 
14. Kent PT. Public health mycobacteriology. A guide for the level III laboratory. Atlanta: Centers for Disease Control and Prevention; 1985.

15. WHO treatment guidelines for drug-resistant tuberculosis. 2016 update. https://www.who.int/tb/areas-of-work/drug-resistanttb/types/en/. last access: 2019/9/25.

16. Dodd PJ, Gardiner E, Coghlan R, Seddon JA. Burden of childhood tuberculosis in 22 high-burden countries: a mathematical modelling study. Lancet Glob Health. 2014;2(8):e453-9.

17. Perez-Velez CM, Marais BJ. Tuberculosis in children. N Engl J Med. 2012;367(4):348-61.

18. Zignol M, Sismanidis C, Falzon D, Glaziou P, Dara M, Floyd K. Multidrug-resistant tuberculosis in children: evidence from global surveillance. Eur Respir J. 2013;42(3):701-7.

19. Wang HJ, Qu D, Dolma L, Zhang R. Epidemiological Survey of Tuberculosis in Residents of 15 Years Old and Above in Nyingch. Journal of Preventive Medicine Information. 2014;30(2):150-3.

20. Ren PZ. Ji. M. Research on the Comprehensive Appraisal for Regional Difference of Economic Development Level in Sichuan Province. JOURNAL OF SICHUAN NORMAL UNIVERSITY(NATURAL SCIENCE). 2007; 30(1):102-105.

21. Organization WH. Addressing Poverty in TB Control. Options for National TB Control Programmes (WHO/HTM/TB/2005.352). 2005.

22. Marais BJ, Gie RP, Schaaf HS, Hesseling AC, Obihara CC, Starke JJ, Enarson DA, Donald PR, Beyers N. The natural history of childhood intra-thoracic tuberculosis: a critical review of literature from the pre-chemotherapy era. Int J Tuberc Lung Dis. 2004;8(4):392-402.

23. Kompala T, Shenoi SV, Friedland G. Transmission of tuberculosis in resource-limited settings. Curr HIV/AIDS Rep. 2013;10(3):264-72.

24. Basu Roy R, Whittaker E, Seddon JA, Kampmann B. Tuberculosis susceptibility and protection in children. Lancet Infect Dis. 2019;19(3):e96-108.

25. WHO. Recommendations for investigating contacts of persons with infectious tuberculosis in low-and middle- income countries. Geneva: The Organization; 2012.

26. Hawn TR, Day TA, Scriba TJ, Hatherill M, Hanekom WA, Evans TG, Churchyard GJ, Kublin JG, Bekker LG, Self SG. Tuberculosis vaccines and prevention of infection. Microbiol Mol Biol Rev. 2014;78(4):650-71.

27. Kaufmann SH. Future vaccination strategies against tuberculosis: thinking outside the box. Immunity. 2010;33(4):567-77.

28. Xu JW, Kuang SS, Wang Q. Study of immunization coverage and influence factors of left-behind children in rural areas of Chongqing. Journal of Tropical Medicine. 2015;10(10):1402-5.

29. Prajapati S, Upadhyay K, Mukherjee A, Kabra SK, Lodha R, Singh V, Grewal HM, Singh S, Delhi Pediatric TBSG. High prevalence of primary drug resistance in children with intrathoracic tuberculosis in India. Paediatr Int Child Health. 2016;36(3):214-8.

30. Macias Parra M, Kumate Rodriguez J, Arredondo Garcia JL, Lopez-Vidal Y, Castanon-Arreola M, Balandrano S, Rastogi N, Gutierrez Castrellon P. Mycobacterium tuberculosis Complex Genotype Diversity and Drug Resistance Profiles in a Pediatric Population in Mexico. Tuberc Res Treat. 2011; 2011:239042.

31. Kim HJ, Yoon HH, Eun BW, Ahn Y, Ryoo S, Kim HJ. The Rate of Drug-Resistant Tuberculosis in Korean Children and Adolescents Since 2007. J Korean Med Sci. 2017;32(6):954-60.

32. Nelson LJ, Schneider E, Wells CD, Moore M. Epidemiology of childhood tuberculosis in the United States, 1993-2001: the need for continued vigilance. Pediatrics. 2004;114(2):333-41.

33. Diande S, Sangare L, Kouanda S, Dingtoumda BI, Mourfou A, Ouedraogo F, Sawadogo I, Nebie B, Gueye A, Sawadogo LT, et al. Risk factors for multidrug-resistant tuberculosis in four centers in Burkina Faso, West Africa. Microb Drug Resist. 2009;15(3):217-21.

34. Brewer TF, Choi HW, Seas C, Krapp F, Zamudio C, Shah L, Ciampi A, Heymann SJ, Gotuzzo E. Self-reported risks for multipledrug resistance among new tuberculosis cases: implications for drug susceptibility screening and treatment. PLoS One. 2011;6(10):e25861. 
35. Respiratory group SOP. Chinese Medical Association: Guidelines for the rational use of antibiotics in acute respiratory infection (Trial) (2nd part). Chin J Pediatri. 2001; 39(6):550-556.

36. Xu YH, Hu ZY. Analysis and Study on drug resistance of 200 random clinical Mycobacterium isolates in Sichuan. Chin J Microbiol Immunol. 2012;32(6):555-60.

37. van der Laan LE, Schaaf HS, Solomons R, Willemse M, Mohamed N, Baboolal SO, Hesseling AC, van Toorn R, Garcia-Prats AJ. Probable Levofloxacin-associated Secondary Intracranial Hypertension in a Child With Multidrug-resistant Tuberculosis. Pediatr Infect Dis J. 2016;35(6):706-8.

38. Sousa J, Alves G, Fortuna A, Falcao A. Third and fourth generation fluoroquinolone antibacterials: a systematic review of safety and toxicity profiles. Curr Drug Saf. 2014;9(2):89-105.

39. Thee S, Garcia-Prats AJ, Donald PR, Hesseling AC, Schaaf HS. Fluoroquinolones for the treatment of tuberculosis in children. Tuberculosis (Edinb). 2015;95(3):229-45.

\section{Figures}

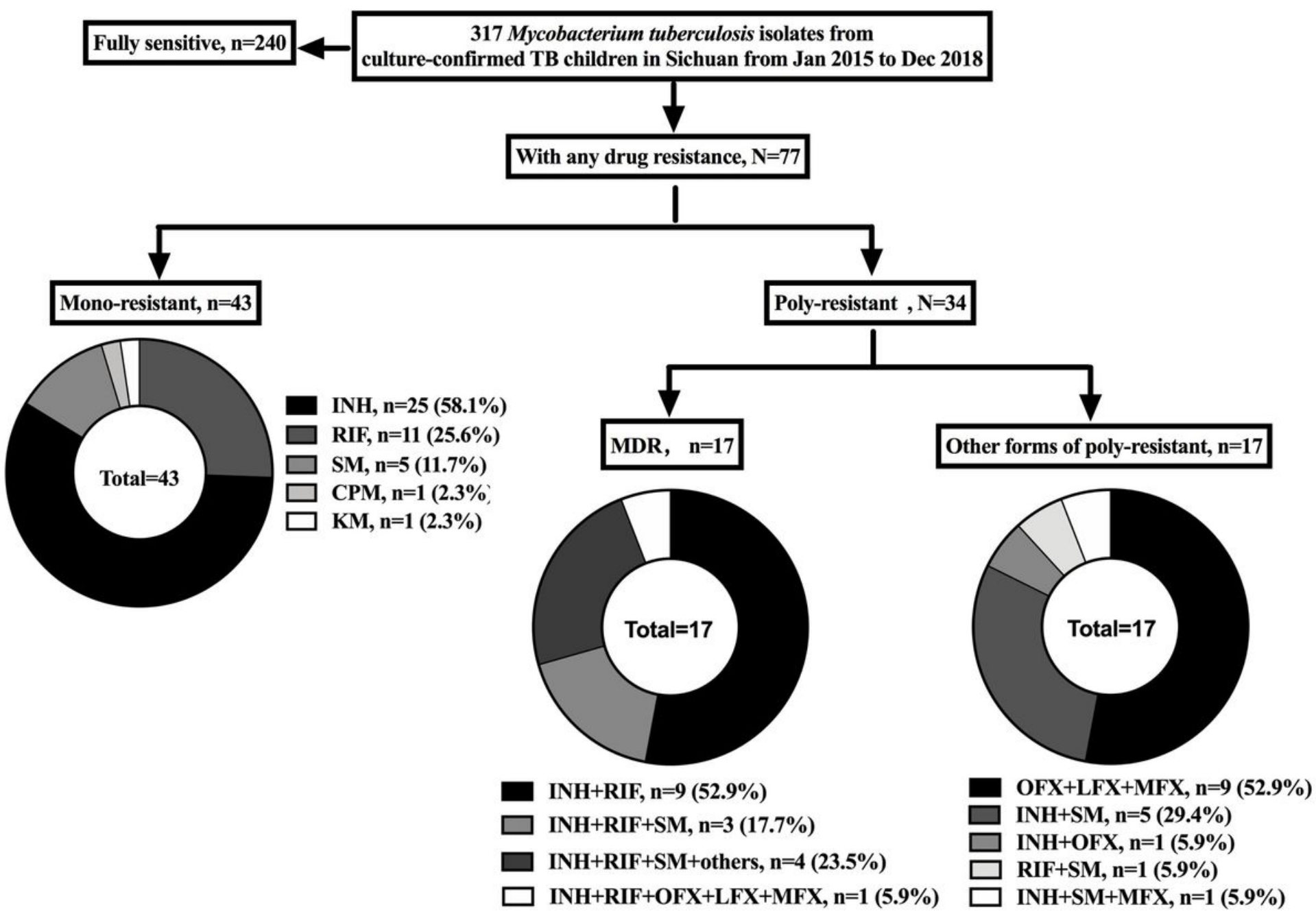

Figure 1

The profile of drug resistant of 317 mycobacterium tuberculosis isolates from culture-confirmed TB children in Sichuan from Jan 2015 to Dec 2018. Abbreviations: RIF, rifampicin; INH, isoniazid; SM, streptomycin; EMB, ethambutol; OFX, ofloxacin; MFX, moxifloxacin; LFX, levofloxacin; AMK, amikacin; KM, kanamycin; and CPM, capreomycin. 